\title{
Use molecular techniques as an alternative tool for diagnosis and characterization of Theileria equi
}

\author{
M.A. El-Seify ${ }^{1}$, N.M. Helmy ${ }^{2}$, N.M. Elhawary ${ }^{1}$, Sh.S. Sorour ${ }^{1}$ and A.M. Soliman ${ }^{2 *}$ \\ ${ }^{1}$ Parasitology Department, Faculty of Veterinary Medicine, Kafrelsheikh University, 33516 Kafrelsheikh, Egypt, \\ ${ }^{2}$ Biotechnology Department, Animal Health Research Institute, Dokki12618, Egypt \\ *Corresponding Author: ahmed.mahmoud8933@yahoo.com
}

(Received September 23, 2017; Accepted October 14, 2017)

\begin{abstract}
The purpose of this study was to determine the prevalence of clinical, subclinical and chronic infection with the equine parasite T. equi in some Egyptian localities (Cairo and Giza governorates). A panel of 396 equine blood samples representing 141 horses, 250 donkeys and 5 mules was collected from equines during the period from April 2015 to March 2016 using microscopic examination and conventional PCR. Microscopically a twenty two (5.56\%) of 396 were positive for $T$. equi merozoites that appeared as small rounded, pyriform shaped and maltase cross shaped merozoites. Among 8/141(5.67\%) horses and 14/250 (5.60\%) donkeys were found to have positive for T.equi. A one hundred blood samples (45 horses, 50 donkeys and 5 mules) selected randomly were also examined by PCR. The results of PCR showed 30/100(11/45 (24.4\%) horses, $18 / 50(36 \%)$ donkeys and $1 / 5(20 \%)$ mule) were positive for T.equi. When the sequenced PCR amplicons $(\mathrm{n}=3)$ were aligned to the reference nucleotide sequences of $T$. equi accessed in Genbank, the horse isolate showed insertion of Thymine (T) base at position 23 and substitution of Thymine (T) base with Cytosine (C) base at position 91, while the donkey and mule isolates have no alterations when compared to the reference sequences. The phylogenetic analysis showed that the sequenced PCR isolates belonged to T.equi. The obtained sequences were deposited in the GeneBank database under accession numbers MF192854, MF192855 and MF192856.
\end{abstract}

Keywords: Equine, T. equi, prevalence, PCR, Egypt Available online at http://www.vetmedmosul.org/ijvs

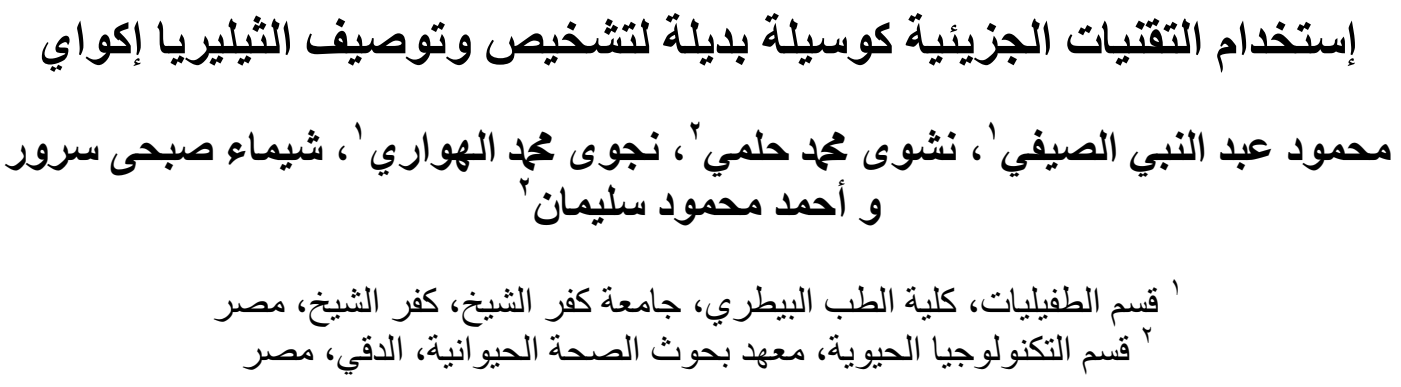

لغرض من هذه الدراسة هو تحديد معدل الخمج بطفيل الثبلريا الخيلية في بعض محافظات جمهورية مصر العربية (القاهرة والجيزة).

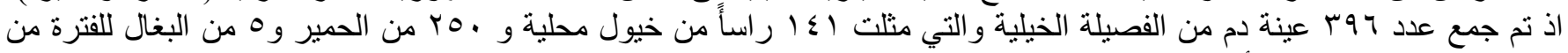

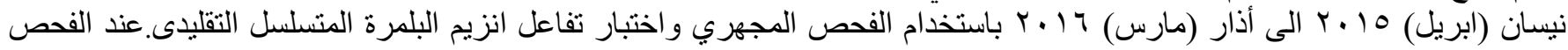

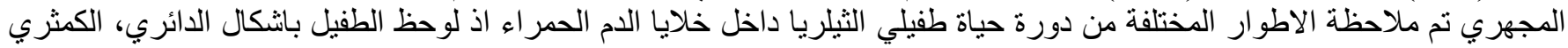

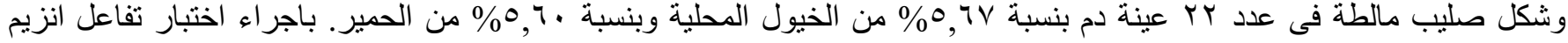

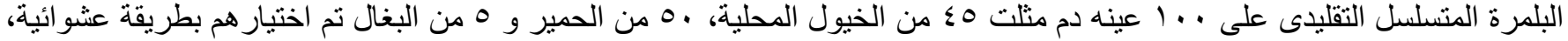

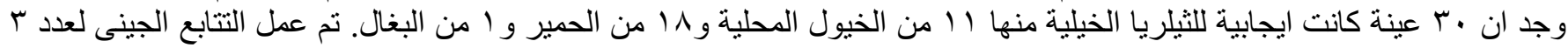




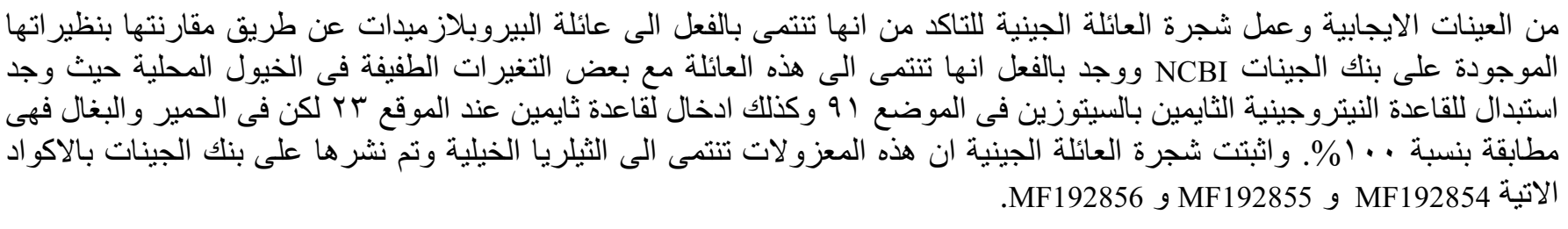

\section{Introduction}

Equine theileriosis is a tick-borne disease of horses, donkeys, mules and zebras with worldwide distribution that affects on equine industry, causing economic losses and significantly impairing the international movement of equines (1). The disease is endemic in tropical, subtropical and some temperate areas of the world (2). It is caused by an obligatory intraerythrocytic protozon of the phylum Apicomplexa which is $T$. equi $(2,3)$. The prevalence of $T$. equi mainly depends on the prevalence of the tick vector which belongs to the genera Boophilus, Dermacentor, Hyalomma and Rhipicephalus $(4,5)$. Other sources of infection are infected blood transfusion and the reusing of infected syringes and surgical instruments (6). T. equi is responsible for the appearance of most clinical cases and causes severe clinical signs (7). Infected equines may remain carriers of $T$. equi for their lifetime. These carrier animals act as a reservoir of infection and as a source of infection for the tick vector $(8,9)$. The spread of the disease is affected by many factors such as climatic conditions and the international movement of equines, so disease-free countries should direct appropriate control strategies when they import equines $(5,10)$. Usually serological tests are used to supervise the movement of horses across borders $(5,11)$.

The prevalence of T. equi was detected in Egypt using microscopical and serological examination. Microscopical examination of stained blood smears has been the standard diagnostic technique for equine theileriosis for several years but it is effective only in acute phase of the infection. In the case of carrier animals, it is not sensitive to detect the infection due to low parasitemia (12). Many serological assays have been developed for the detection of antibodies against $T$. equi such as immunofluorescent antibody technique (IFAT), immunochromatographic test, complement fixation test (CFT) and enzyme-linked immunosorbent assay (ELISA) (13). These methods have proved to be more sensitive although they have some disadvantages related to antibody detection limit and crossreactivity to other Babesia species (14). Polymerase chain reaction (PCR) proved to has higher sensitivity and specificity in detection of the protozoal DNA of Theileria species $(15,16)$. The PCR technique has sufficient sensitivity to detect protozoal DNA from a blood sample with parasitemia of $0.000001 \%$ (15). The aim of this study was to determine the prevalence of $T$. equi in the equine population in both two Egyptian governorates (Cairo and Giza) based on microscopic examinations and molecular techniques. Further, molecular characterization of $T$. equi was made based on $18 \mathrm{~s}$ rRNA sequences.

\section{Materials and methods}

\section{Sample collection and microscopic examination}

A total number of 396 equine blood samples representing 141 horses, 250 donkeys and 5 mules was collected from different localities in Egypt (Cairo and Giza), during the period from April 2015 to March 2016. All samples were collected by the jugular venipuncture method using EDTA-tubes from working equines. Some of these animals was healthy and others were clinically diseased. All tubes were marked by the necessary data (age, sex, location and date of collection) and then samples were sent in an ice box to the Parasitology Department, Faculty of Veterinary Medicine, Kafrelshiekh University, for examination. In the lab, thin blood films were prepared from the whole blood, stained with Giemsa stain. and examined for the presence of $T$. equi merozoites using an oil emersion lens of the light microscope according to (17).

\section{DNA extraction and PCR amplification}

Genomic DNA was extracted from 100 whole blood samples (45 horses, 50 donkeys and 5 mules) representing microscopically positive blood samples $(n=22)$ and from randomly selected microscopically negative blood samples $(\mathrm{n}=78)$, using Thermo Scientific ${ }^{\mathrm{TM}}$ GeneJET Genomic DNA Purification Kit (Cat No \#K0722) according to the instructions in the manufacturer's manual. The obtained DNA was stored at $-20^{\circ} \mathrm{C}$ until used in the downstream applications. The PCR technique was applied using primers developed by (15) that specifically detect $392 \mathrm{bp}$ from the $18 \mathrm{~s}$ rRNA gene of T.equi. The assay utilized a universal forward primer (Bec-UF2) with a sequence 5TCGAAGACGATCAGATACCGTCG-3 and a Theileria equi specific reverse primer (Equi-R) with a sequence 5TGCCTTAAACTTCCTTGCGAT-3. PCR was performed using GoTaq ${ }^{\circledR}$ G2 Flexi PCR Kit (Promega, USA) with a total volume of $25 \mu \mathrm{l}$ containing $5 \mathrm{X}$ Green GoTaq ${ }^{\circledR}$ Flexi Buffer $(10 \mu \mathrm{l}), 25 \mathrm{mM} \mathrm{MgCl} 2$ Solution $(2 \mu \mathrm{l})$, PCR Nucleotide Mix (dNTPs) $10 \mathrm{mM}$ each $(1 \mu \mathrm{l})$, Primer mix 10 pmol $(1 \mu 1), 1.25$ u GoTaq ${ }^{\circledR G} 2$ Flexi DNA Polymerase 
template DNA $(5 \mu 1)$, DNase/RNase free water $(7 \mu 1)$. The thermal profile was $95^{\circ} \mathrm{C}$ for $5 \mathrm{~min}$, followed by 35 successive cycles of denaturation at $96^{\circ} \mathrm{C}$ for $1 \mathrm{~min}$, annealing at $60^{\circ} \mathrm{C}$ for $1 \mathrm{~min}$, and extension at $72^{\circ} \mathrm{C}$ for 1 min. Then a final extension was made at $72^{\circ} \mathrm{C}$ for $5 \mathrm{~min}$, and then holding stage at $4{ }^{\circ} \mathrm{C}$ for infinite time. $8 \mu 1$ of the generated PCR products were migrated on $1.5 \%$ ethidium bromide stained agarose gel under a constant volt of $80 \mathrm{~V}$ for $40 \mathrm{~min}$. The gel was then visualized using UVTransilluminator and then photographed by the associated camera (15). T. equi positive samples showed a band of 392 bp.

\section{Sequencing and phylogenic analysis}

Positive PCR products $(n=3)$ representing 1 horse, 1 donkey and 1 mule were extracted from agarose gel using a thermo scientific gene JET gel extraction kit (Cat No. K0691) and were sent to the sequencing unit at the Animal Health Research Institute, Dokki, Giza for sequencing in a single direction using specific $T$. equi reverse primer (5TGCCTTAAACTTCCTTGCGAT-3). The sequences were compared with each other and then with the GenBank database by the nucleotide sequence homology search. using the BLAST analysis database that is available at the National Centre for Biotechnology Information (NCBI) (http://blast.ncbi.nlm.nih.gov). All sequence data were edited subsequently by the naked eye, using Bioedit 7.2.5 software (http://www.mbio.ncsu.edu/BioEdit/bioedit.html). A phylogenetic analysis was performed using MEGA version 7 (http://www.megasoftware.net). A phylogenetic tree was produced by applying the Neighbor-Joining technique with using Hepatozoon canis (DQ439543.1) as out groups. Sequences produced during this study have been deposited in the GeneBank database under accession numbers MF192854, MF192855 and MF192856.

\section{Results}

A total number of $22(5.56 \%)$ of the 396 Giemsa-stained blood smears were harbored T. equi merozoites (Table 1). They appeared as small rounded, pyriform shaped and maltase cross shaped merozoites (Figure 1).

The conventional PCR amplification showed that a total number of $30(30 \%)$ out of 100 examined blood samples showed $392 \mathrm{bp}$ fragments in $1.5 \%$ ethidium bromide agarose gel which were specific for $T$. equi (Figure 2). The infection rate of blood samples tested by conventional PCR amplification was compared with the infection rate of the same samples found by microscopical examination, as shown in Table 2.

When the sequenced PCR amplicons $(\mathrm{n}=3)$ were aligned to the reference nucleotide sequences of $T$. equi accessed in the Genbank, the horse isolate showed the insertion of Thymine $(\mathrm{T})$ base at position 23 and substitution of
Thymine (T) base with Cytosine (C) base at position 91; while the donkey and mule isolates had no alterations when compared to the reference sequences (Figure 3). The phylogenetic analysis showed that the sequenced PCR isolates belong to T. equi (Figure 4).

Table 1: Prevalence of T. equi among examined animals based on microscopical examination of Giemsa-stained blood smears

\begin{tabular}{lccc}
\hline & $\begin{array}{c}\text { No. } \\
\text { examined }\end{array}$ & $\begin{array}{c}\text { No. } \\
\text { infected }\end{array}$ & $\begin{array}{c}\text { Infection rate } \\
(\%)\end{array}$ \\
\hline Horses & 141 & 8 & 5.67 \\
Donkeys & 250 & 14 & 5.60 \\
Mules & 5 & -- & -- \\
\hline Total & 396 & 22 & 5.56 \\
\hline
\end{tabular}

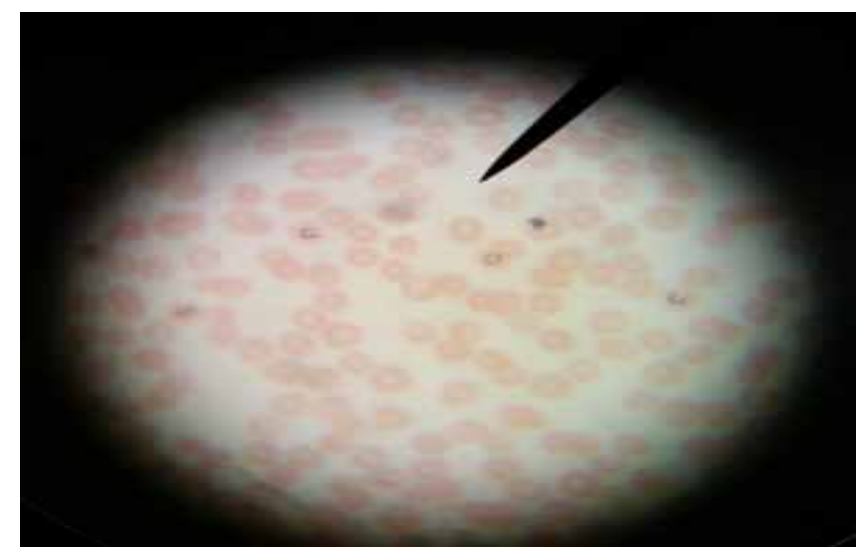

Figure 1: Microscopically stained blood smears showed small rounded merozoites of Theileria equi (x100).

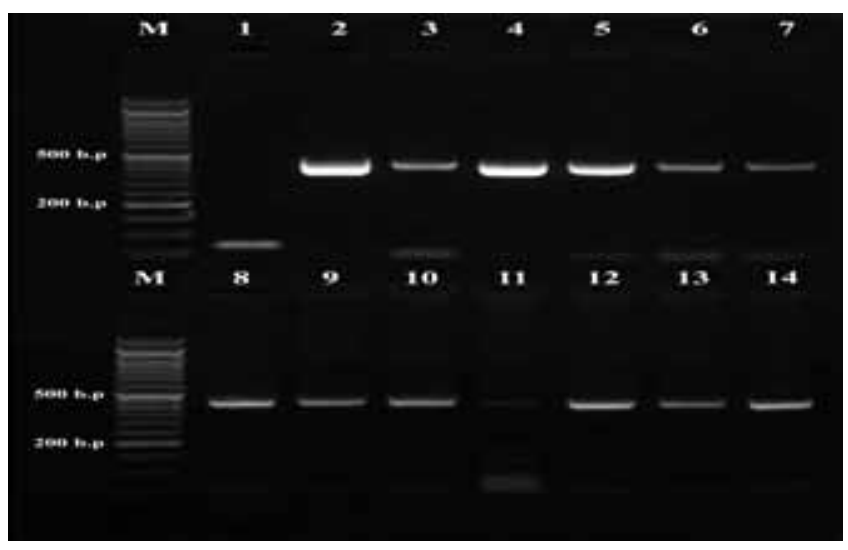

Figure 2: Conventional PCR detection of Theileria equi at $392 \mathrm{bp}$ fractionated on $1.5 \%$ agrose gel. Lane M: $50 \mathrm{bp}$ DNA ladder, Lane 1: T. equi negative control, Lane 2: $T$. equi positive control (392bp), Lane 3-14 T. equi positive samples (392bp). 
Iraqi Journal of Veterinary Sciences, Vol. 32, No. 1, 2018 (5-11)

MF 192855 , Theileris equi wie $E$ WF 192856 - Theller1a_equi_donkey

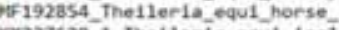
0227629.1 Theileris_equi_1so1 10227623.1 Thelleria_equi_iso1 DC696428, 1_Thelleria_equi_iso1 nuzs9e96.1_The11er1s_equi_1so1 KT 307980 . 1 B Babesis_equi_iss_r-1 LCees132.1.8abesis_equi, Eene-f AAS15310.1_Babesis_equi sene_f KY46.036.1.Thelleris_equi_clon osa19520.1 Babesis_equi_tsolat 0wat6922.2 Babesis equil isolat au240071.1.Theileris_equi_clan FU642512.1 Robesle cabal11 ien

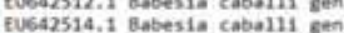
AFBa1135.1 Thelleris lestoquar twoasso0.1 Theileria annulata De439543, 1 Hepatozoon cants is

MF192855_The1leria_equi_mule,E MF192856_thellerta_equi_donkey MF 192855 _Theller-10_equi_horse ×227623 1 Thelleria equi 1 isol OBB96428.1 Theller1a equi_ 1901 kuzb9996.1 Thelleria_equi isol KT307989,1_Babesia_equi_185 - -1

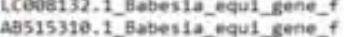
215105.1.8.equi_sene_encoding.

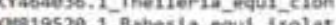
0619520.1_Bobesis_equi_isolat 06046922.1.8abesia_equi_Llsolat Kuzc0071.1_Thelleria_equi_clion EU642512.1 Babesia Cabal11 gen
EUGA2514.1 Babesis cabal11 gen

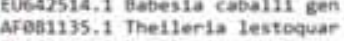
Eves36e日. 1 Thelleris annulat: pe439543.1 Mepatozoon canis is

MF 192855 -Thelleris_equi_wle,t nF 192856 -Thelleria_equi_donkey Wr192854 Thelleris equi horse 0027623 . The Theris_equi_isol

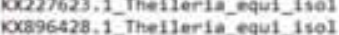
Ku289428.1 Theileria_equi_isol Ku2 19096.1 Theileris_equi_isal

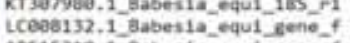

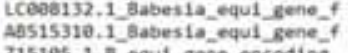
215105.1 . D.equi gene-encoding.

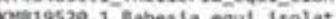
ow owa6s2 .1

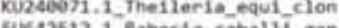
KuG 42512.1 gabesis cabalit gen cuka 2514.1 tabesia cabaili gen Arosis3s.1 Therieria lestoquar

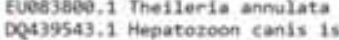

MF 192855 _theller1s_equi_wile $t$ MF 192856 thellerid_equi_donkey MF 192854-Thelleris_equi horseDa27623.1 Thelleria-equi_iso1 100696428.1 Thelleria_equi_isol kuza9096.1 Thelleria_equi_iso1

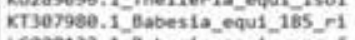
LCees132.1 labesia_equi_ene-t

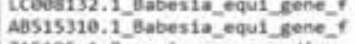
zistes.1 B.equi gene_encoding

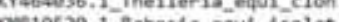
ost ONate922.1. Kundeari.2 mellerin_equi_cloir EU642514.1 Babesila catall1 gem AFab1135.1 Theslierta lestoquar Eues3see. 1 Theller1s annulata
po439543.1 thepatozoon canis is

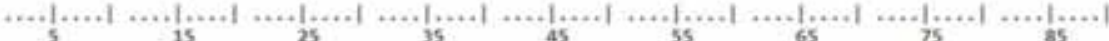

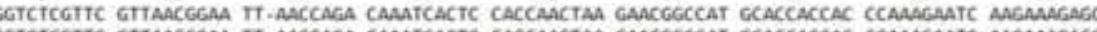

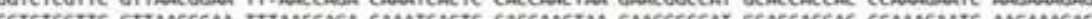

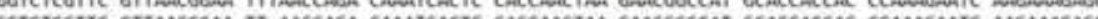

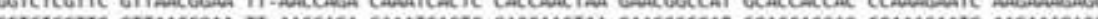

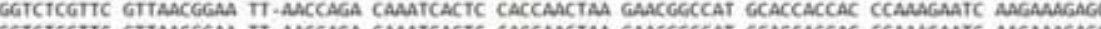

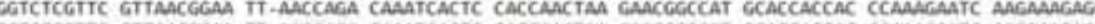

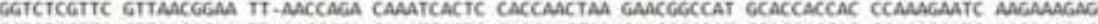

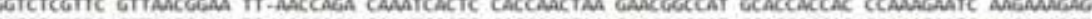

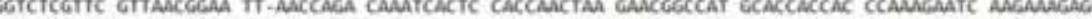

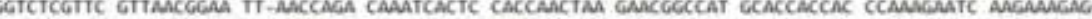

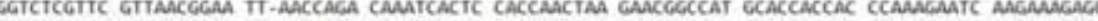

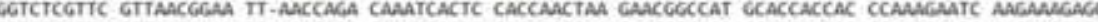

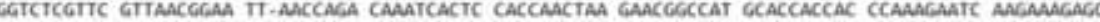

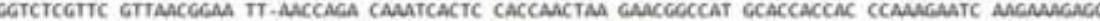

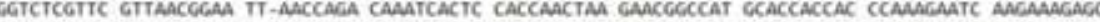

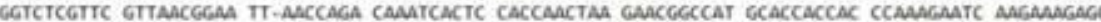

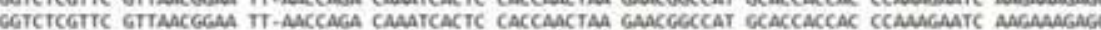

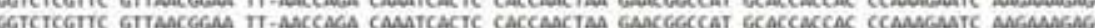

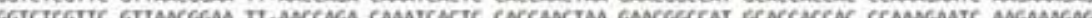

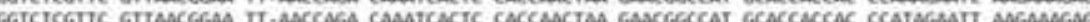

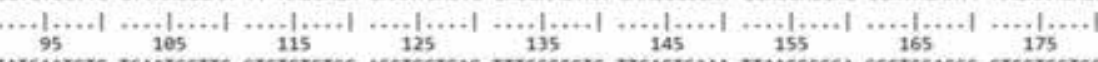

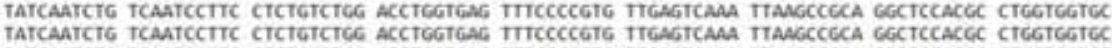
CATCAATCTG TCAATCCTIC CTCTGTCTOG ACCTGGTCAG TITCCCCGTG TTEAGTCAAA TTAAGCCCLA GGCTCCACGC CTGGTGSTUE

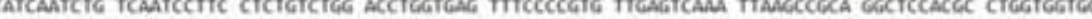

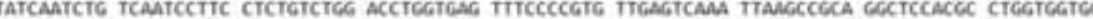
PATCAATCTG TCAATCCTIC CTCTGTCTGG ACCTGGTGAG TITCCCCGTG TTGAGTCAMA TTAAGCCGCA GGCTCCACGC CTGGTGGTC

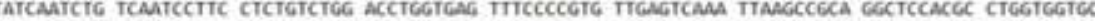

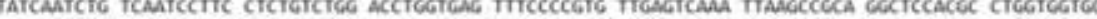
PATCAATCTG TCAATCCTIC CTCTGTCTGG ACCTCGIGAS TITCCCCGTG TIGAGTCAAA TIAACCCLCA CGCTCCACEC CTGGTGGTC TATCAATCTG TCAATCCTTC CTCTGTCTGG ACCTGGTAG TITCCCCGTG TTGAGTCAAA TTAAGCCECA GGCTCCACGC CTGGTEGTCE

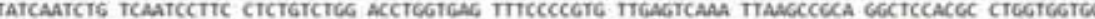

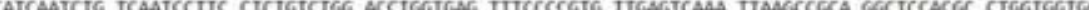

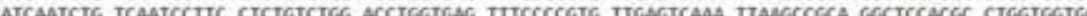

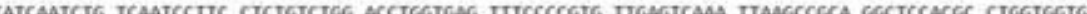

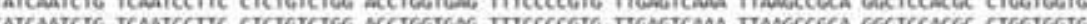

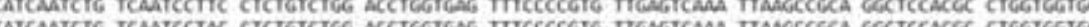

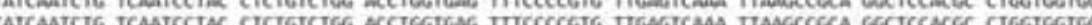

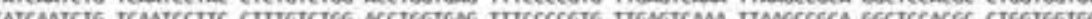

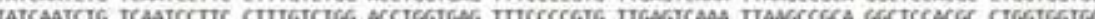

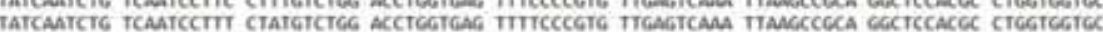

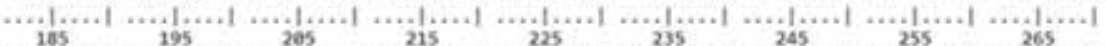

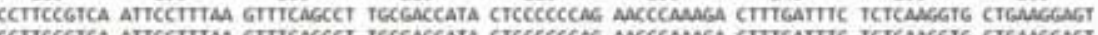

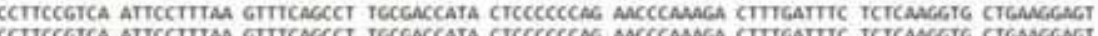

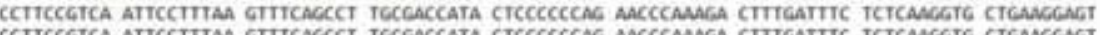

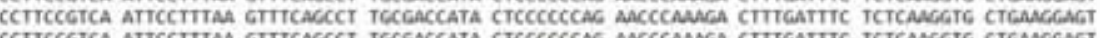
CCTICCGTCA ATTCCTITAA GTTTCACCCT TECGACCATA CTCCCCCCAG AACCCMAAGA CTITGATTTC TCTCAAGGTG CTGANCGAGT CCITCCGTCA ATTCCITTAA GITICACCCT TECGACCATA CTCCCCCCAG AACCCANGA CTITGATITC TCTCANGGTG CTGANGGAGT CCITCCGTCA ATTCCIITAA GITTCACCCT TECGACCATA CTCCCCCCAC AACCCMAAGA CTITGATTIC TCTCAAGGTG CTGANGGAGT

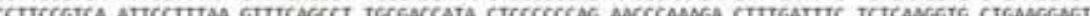

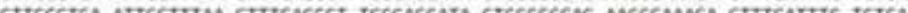

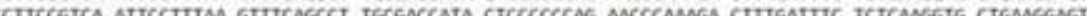

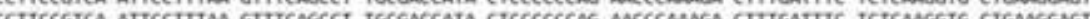

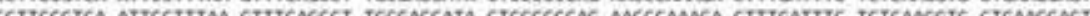

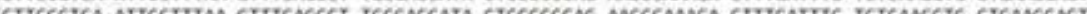

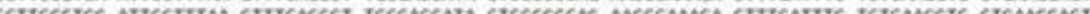

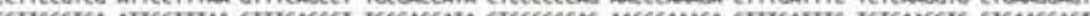

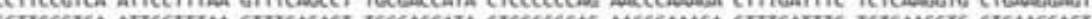

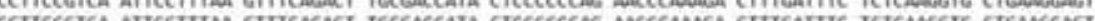

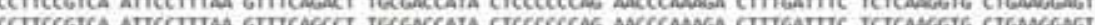

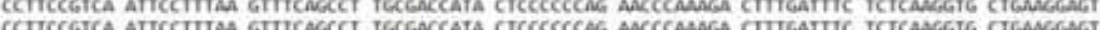

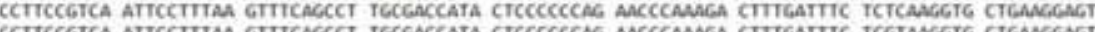

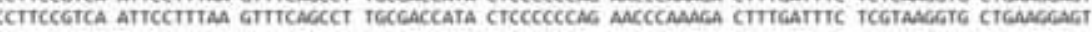

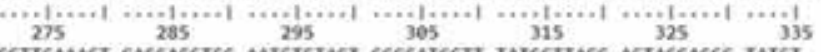

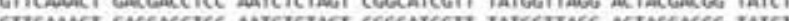
CIITCANAC GACGACCTCC AATCFCTAGT CGECATCGTI TATGGTTAGG ACTACGACGG TATCY GGTCAAACT GACGACCTCC AATCTCTAGT CGGCATCGIT TATGGTTAGG ACTACGACGG TATCT CGITCALACT GACGACCTCC AAFCTCTAGT CGECATCGII TATGGITAGG ACTACGACGG TATCY CGITCAAACT GACGACCTCC AATCTCTAGT CGGCATGGT TATGGTTACG ACTACGACGO TATCF CGITCAAACT GACGACCTCC AATCTCTAGT CGGCATCGT TATGGTTAGG ACTACGACGG TATCF CGITCANACT GACGACCTCC AATCTCTAGT CGGCATCGT TATGGTTAGG ACTACGACGG TATCT GTICAAACT GACGACCTCC AATCTCTAGT CGCCATCGIT TATGGTTASG ACTACGACGG TATCT CGITCANACT GACGACCTCC AATCTCTAGT CGGCATCGIT TATGGITAGG ACTACGACGG TATC CGTTCAAACT GACGACCTCC AATCTCTAGT CGECATCGIT TATGGTTACG ACTACGACGG TATCT

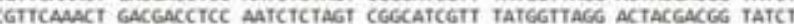
CGITCAaACT GaCGACCTCC AATCTCTAGT CGECATCGIT TATGGTIAGG ACTACGACGG TATCT GTTCAAACT GACGACCTCC AATCTCTAGT CGECATCGTT TATGGTTACS ACTACGACES TATCT CGTICAAACT GACGACCTCC AATCTCTAGT CGGCATCGTI TATGGIIASG ACTACGACGG TATCT CGTTCAMACT GaCGACCTCC AATCTCTAGT CGSCATCGT TATAGTTAGG ACTACGACCG TATCT

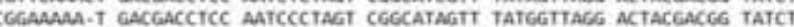
CACCAAAA-T GACGACCTCC AATCCCTAGT CGGCATAGIT TATGGTIAGG ACTACGACGG TATCI

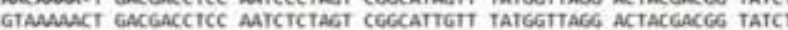
CGTAAAACT GACGACCTCC AATCTCTAGT CGGCATAGTT TATGGTTACG ACTACGACGG TATCI

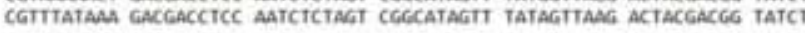

Figure 3: Alignment of nucleotide sequence of Theileria equi of isolates obtained from Egyptian equines with reference to sequences of Theileria equi accessed in the genbank, in addition to sequences of Hepatozoon canis as out group. 
Table 2: Infection rates of 100 tested blood samples for T. equi using microscopical examination compared with conventional PCR amplification

\begin{tabular}{llcccc}
\hline Animals & Number & \multicolumn{2}{c}{ Microscopical examination } & \multicolumn{2}{c}{ Conventional PCR examination } \\
\cline { 2 - 6 } & examined & Number infected & Infection rate (\%) & Number infected & Infection rate (\%) \\
\hline Horses & 45 & 8 & 17.8 & 11 & 24.4 \\
Donkeys & 50 & 14 & 28 & 18 & 36 \\
Mules & 5 & -- & -- & 1 & 20 \\
\hline Total & 100 & 22 & 22 & 30 & 30 \\
\hline
\end{tabular}

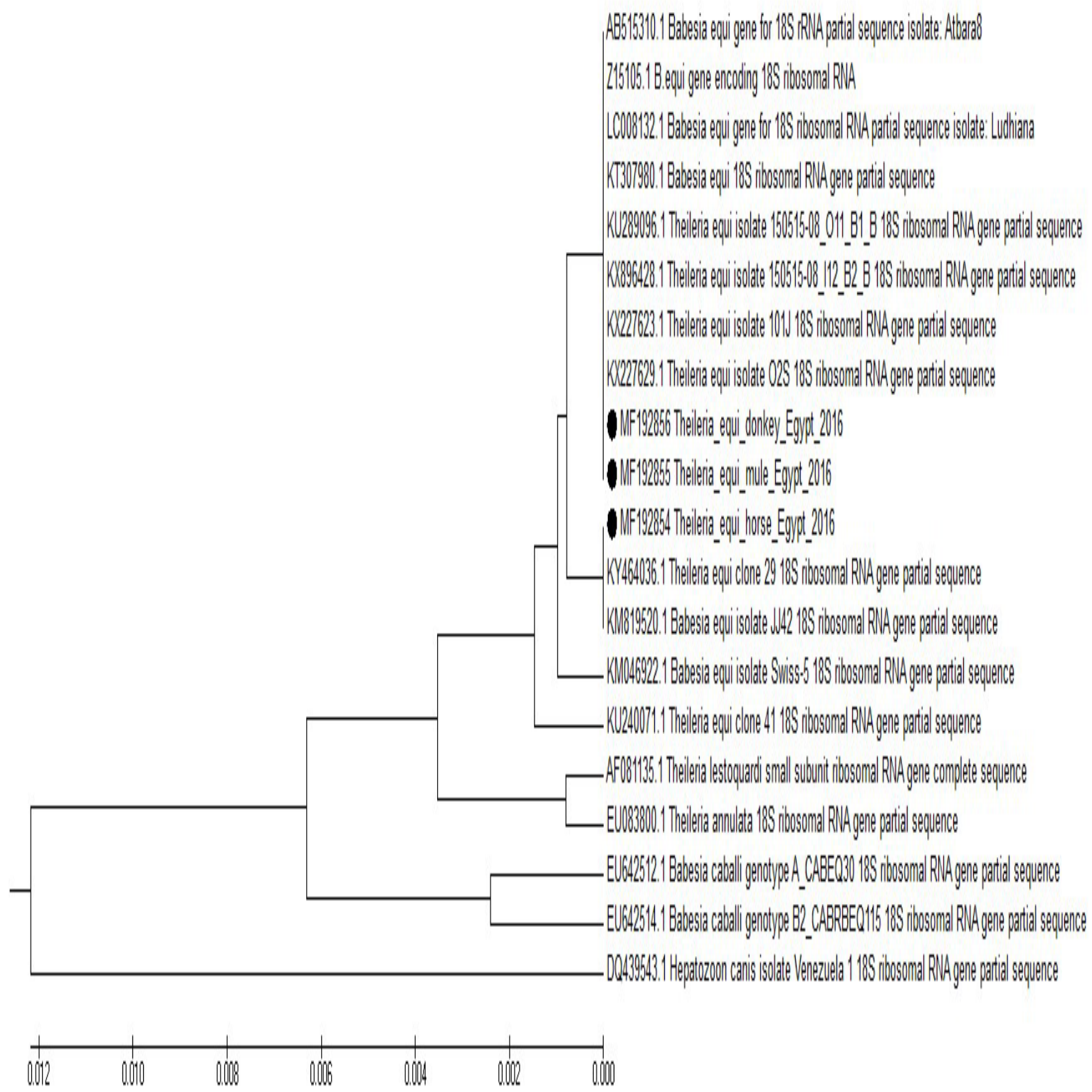

Figure 4: Genetic relationship of Theileria equi isolates obtained from egyptian equines with reference to sequences of Theileria equi accessed in the genbank. Phylogenetic tree was produced by applying Neighbor-Joining technique of the nucleotide sequence of the 18s rRNA gene with using Hepatozoon canis (DQ439543.1) as out group. 


\section{Discussion}

The objective of this study was to estimate the prevalence of $T$. equi microscopically and by conventional PCR and then to apply the molecular characterization of $T$. equi to specific geographic areas in Egypt (Cairo and Giza governorates).

Microscopic examination has been shown to be insensitive to detect low parasitemia especially in areas where the disease is endemic (18). More-ever, microscopic examination depends on host specificity and this is less useful with such parasites of broader host specificity as Babesia microti (19). The conclusion is that, molecular techniques are a more objective tool for the diagnosis of $T$. equi (20).

In this study, the overall prevalence of $T$. equi by microscopical examination of blood samples was $(5.56 \%)$. This result agrees with previous studies in Egypt that showed the infection rate of T. equi is between $5 \%$ and $10 \%$ (21-23). The infection rate found in this study was lower than that recorded earlier in Egypt, which showed infection rates of $19.8 \%, 34 \%, 13.9 \%, 18 \%$ and $38.9 \%$ respectively (24-28). This may be due to differences in the various geographic areas in Egypt and high vector tick activity in the areas of sampling. Lower activity now may be due to a greater awareness by owners of preventive measures, more aggressive treatment of infected animals and increasing efforts of tick control. The time of sampling can make a difference, where samples are collected at the acute or chronic stage of the disease.

The infection rate of $T$. equi was recorded in various other countries, as in Iran 2016, Iran 2014 and in central Ethiopia $(9.7 \%, 9.1 \%$ and $12.2 \%$ respectively) $(7,29,30)$. The prevalence found was higher than that recorded in Egypt, due to such different environmental conditions as temperature and humidity, which affects tick activity. Also, type of animal, whether racing or working equines. Hygienic measures and vector control also play a role in such differences.

In the present study, there are no marked differences between the infection rate of $T$. equi in horses $(5.67 \%)$ and donkeys (5.6\%). This agrees with (30), who recorded infection rates $(51.2 \%$ and $51.6 \%)$ of $T$. equi in horses and donkeys.

In this study, molecular techniques showed a higher sensitivity than microscopic examination in the diagnosis of subclinical and carrier animals and this agrees with $(9,31,32)$.

In the present study, by application of conventional PCR, the $T$. equi infection rate was $30 \%$. This result was lower than that recorded in Egypt by (28) $(77.80 \%)$ and higher than $(29,35)$, who recorded infection rates of $10.83 \%$ and $13.90 \%$ respectively by PCR amplification.
Other countries recorded higher rates of infection, as in Brazil $96 \%$ by nested PCR (34), Egypt $47.7 \%$ by nested PCR (26) and Iran $96.8 \%$ by conventional PCR (29). The lower prevalence was recorded in (35), Brazil (15.0\%) (36) and Turkey (2.96\%) (37).

Sequencing and phylogenetic analysis of piroplasms depends mainly on $18 \mathrm{~s}$ rRNA, due to its low substitution rate; constrained and conserved function and occurrence in multiple copies (38). Sequencing and phylogenetic analysis of the positive PCR product for T. equi recorded a $100 \%$ similarity with previously published sequences on the GeneBank database for donkey and mule sequences, but a $99 \%$ similarity for horse sequences, as illustrated in figure ( 4 ).

In conclusion, it is recommended to use PCR as a rapid confirmatory technique for $T$. equi because it has higher sensitivity than microscopic examination in subclinical and chronic phases of the infection and in carrier cases. Also, it is recommended to conduct further studies on T.equi, to determine the best method for diagnosis and to illustrate the best control and preventive strategies against this very significant equine parasite.

\section{References}

1. Zobba R, Ardu M, Niccolini S, Chessa B, Manna L, Cocco R, et al. Clinical and Laboratory Findings in Equine Piroplasmosis. J Equine Vet Sci. 2008;28:301-308.

2. Rothschild CM. Equine Piroplasmosis. J Equine Vet Sci. 2013;33:497-508.

3. Sellon DC LM. Equine piroplasmosis: Equine infectious diseases. Saunders, Missouri. Elsevier. 2007;

4. Bhagwan J, Kumar A, Kumar R, Goyal L, Goel P, Kumar S. Molecular evidence of Theileria equi infection in Hyalomma anatolicum ticks infested on sero-positive Indian horses. Acta Parasitol. 2015;60:322-329.

5. Guidi E, Pradier S, Lebert I. Piroplasmosis in an endemic area: analysis of the risk factors and their implications in the control of Theileriosis and Babesiosis in horses. Parasitol Res. 2015;114:71-83.

6. Farkas R, Tánczos B, Gyurkovszky M, Földvári G, Solymosi N. Veterinary Parasitology Serological and molecular detection of Theileria equi infection in horses in Hungary. Vet Parasitol. 2013;192:143-148.

7. Malekifard F, Tavassoli M, Yakhchali M, Darvishzadeh R. Detection of Theileria equi and Babesia caballi using microscopic and molecular methods in horses in suburb of Urmia, Iran. Vet Res Forum. 2014;5:129-133.

8. Rüegg SR, Torgerson P, Deplazes P, Mathis a. Age-dependent dynamics of Theileria equi and Babesia caballi infections in southwest Mongolia based on IFAT and/or PCR prevalence data from domestic horses and ticks. Parasitol. 2007;134:939-947.

9. Heim A, Passos LMF, Ribeiro MFB, Costa LM, Bastos C V., Cabral $\mathrm{DD}$, et al. Detection and molecular characterization of Babesia caballi and Theileria equi isolates from endemic areas of Brazil. Parasitol Res. 2007;102:63-68.

10. Sigg L, Gerber V, Gottstein B, Doherr MG, Frey CF. Seroprevalence of Babesia caballi and Theileria equi in the Swiss horse population. Parasitol Int. 2010;59:313-317.

11. Sgorbini M, Bonelli F, Nardoni S, Rocchigiani G, Corazza M, Mancianti F. Journal of Equine Veterinary Science Seroprevalence and Molecular Analysis of Babesia caballi and Theileria equi in 
Horses From Central Italy During a 10-Year Period. J Equine Vet Sci. 2015;35:865-868.

12. Quintão-Silva MG, Ribeiro MFB. Infection Rate of Babesia spp. Sporokinetes in Engorged Boophilus microplus from an Area of Enzootic Stability in the State of Minas Gerais, Brazil. Mem Inst Oswaldo Cruz. 2003;98:999-1002.

13. Kim C, Beatriz L, Blanco C, Alhassan A, Iseki H, Yokoyama N, et al. Diagnostic real-time PCR assay for the quantitative detection of Theileria equi from equine blood samples. Vet Parasitol. 2008;151:158-163.

14. BrÜning A, Phipps P, Posnett E, Canning EU. Monoclonal antibodies against Babesia caballi and Babesia equi and their application in serodiagnosis. Vet Parasitol.1997;68:11-26.

15. Alhassan A, Pumidonming W, Okamura M, Hirata H, Battsetseg B, Fujisaki K, et al. Development of a single-round and multiplex PCR method for the simultaneous detection of Babesia caballi and Babesia equi in horse blood. Vet Parasitol. 2005;129:43-49.

16. Davitkov D, Vucicevic M, Stevanovic J, Krstic V, Slijepcevic D, Glavinic U, et al. Molecular detection and prevalence of Theileria equi and Babesia caballi in horses of central Balkan. Acta Parasitol. 2016;61:337-342.

17. Levine. Veterinary Protozoology. Iowa state Univerity Press.Ames.USA. 1982.

18. Calder JAM, Reddy GR, Chieves L, Courtney CH, Littell R, Livengood JR, et al. Monitoring Babesia bovis Infections in Cattle by Using PCR-Based Tests. J Clin Microbiol. 1996;34:2748-2755.

19. Edelhofer R, Kanout A, Schuh M, Kutzer E. Improved disease resistance after Babesia divergens vaccination. 1998;181-187.

20. Persing D, Conrad P. Babesiosis: new insights from pylogenetic analysis. Infect Agents Dis. 1995;4:182-195.

21. Arafa MI. Studies On Ecto And Endoparasites Of Equines In Assiut Governorate. Assiut Univ Vet Med Dep Parasitol Thesis. 1998.

22. El-Fayoumy MM, Iman MB. Seroepidemiological Studies on Theileria Equi(Babesia equi) and Babesia Caballi Infections Among Horses in Egypt. 12th SciCong,FacVetMed,Assuit Univ, Egypt. 2006. pp:251-264.

23. El-Kelesh EAM, Radwan IG., Girgis S, Ibrahim O. Serodiagnosis of equine Piroplasmosis in Egypt using CELISA. Egypt J Agric Res. 2012;90:483-493.

24. Radwan AMM. An Investigation On Parasitic Infection In Equines. Banha Univ. Thesis. 2009.

25. Salib F a., Youssef RR, Rizk LG, Said SF. Epidemiology, diagnosis and therapy of theileria equi infection in giza, egypt. Vet World. 2013;6:76-82.

26. Mahmoud MS, El-ezz NTA, Abdel-shafy S, Nassar SA, Namaky AH El, Khalil WKB, et al. Assessment of Theileria equi and Babesia caballi infections in equine populations in Egypt by molecular, serological and hematological approaches. Paras Vect. 2016;9:1-10.

27. Ibrahim AK, Gamil IS, Abd-El baky AA, Hussein MM, Tohamy AA. Comparative Molecular and Conventional Detection Methods of Babesia equi ( B. Equi ) in Egyptian Equine. Glob Vet. 2011;7:201210.

28. Farah AW, Hegazy NA, Romany MM, Soliman YA, M DA. Molecular detection of Babesia equi in infected and carrier horses by polymerase chain reaction. Egypt J Immunol. 2003;10:73-79.

29. Habibi G, Esmaeilnia K, Hablolvarid MH. Microscopic and Molecular detection of Theileria ( Babesia ) equi infection in equids of Kurdistan Province, Iran. Iran J Parasitol. 2016;11:86-90.

30. Gizachew A, Schuster RK, Joseph S, Wernery R, Georgy NA, Elizabeth SK. Piroplasmosis in Donkeys-A Hematological and Serological Study in Central Ethiopia. J Equine Vet Sci. 2013;33:1821.

31. Salim BOM, Hassan SM, Bakheit MA, Alhassan A, Igarashi I, Karanis P, et al. Diagnosis of Babesia caballi and Theileria equi infections in horses in Sudan using ELISA and PCR. Parasitol Res. 2008;103:1145-1150.

32. Mahdy OA, Nassar AM, Mohamed BS, Mahmoud MS. Comparative Diagnosis Utilizing Molecular and Serological Techniques of Theileria equi Infection in Distinct Equine Populations in Egypt. Internat J Chem Tech Res. 2016;9:185-197.

33. Slivinska K, Víchová B, Werszko J, Szewczyk T, Wróblewski Z, Pet'ko B, et al. Molecular surveillance of Theileria equi and Anaplasma phagocytophilum infections in horses from Ukraine, Poland and Slovakia. Vet Parasitol. 2015;1-14.

34. Battsetseg B, Lucero S, Xuan X. Detection of natural infection of Boophilus microplus with Babesia equi and Babesia caballi in Brazilian horses using nested polymerase chain reaction. Vet Parasitol. 2002;107:351-357.

35. Rampersad J, Cesar E, Campbell MD, Samlal M, Ammons D. A field evaluation of PCR for the routine detection of Babesia equi in horses. Vet Parasitol. 2003;114:81-87.

36. Nizoli LQ, Gotze MM, Felix SR, Silva SS Da, Nogueira CEW. Frequency of seropositive equines for Theileria equi in the Southern Rio Grande do Sul State, Brazil. Parasitol Latinoam. 2008;63:46-50.

37. Kizilarslan F, Yildirim A, Duzlu O, Inci A, Onder Z, Ciloglu A. Molecular detection and characterization of Theileria equi and Babesia caballi in horses (Equus ferus caballus) in Turkey. J Equine Vet Sci 2015;1-21.

38. Hunfeld K, Hildebrandt A, Gray JS. Babesiosis: Recent insights into an ancient disease. Int J Parasitol. 2008;38:1219-1237. 